\title{
Diagnostic Utility of Immunophenotyping by Flow Cytometry for Diagnosis and Classification of Acute Leukemias in Tikur Anbessa Specialized Hospital, Addis Ababa, Ethiopia
}

\author{
Metasebia Tegegen ${ }^{1 *}$, Fatuma Hassen ${ }^{2}$, Abdulaziz Abubeker ${ }^{3}$, Fissehatsion Tadesse ${ }^{3}$, \\ Daniel Hailu ${ }^{3}$, Ashenafi Alemu ${ }^{1}$, Aster Tsageye ${ }^{2}$, Rawleigh Howe ${ }^{1}$, Amha Gebremedhin ${ }^{3}$ \\ ${ }^{1}$ Department of Immunology, Armauer Hansen Research Institute, Addis Ababa, Ethiopia \\ ${ }^{2}$ Department of Medical Laboratory Science, College of Health Science, Addis Ababa University, Addis Ababa, Ethiopia \\ ${ }^{3}$ Department of Haematology, School of Medicine, Addis Ababa University, Addis Ababa, Ethiopia
}

\section{ARTICLE INFO}

Received : 28 July 2020

Reviewed : 24 August 2020

Accepted : 09 March 2021

\section{Keywords:}

ALL, AML, flow cytometry, immunophenotyping, leukemia

\author{
*Corresponding author: \\ Metasebia Tegegen \\ Armauer Hansen Research Institute \\ Addis Ababa, Ethiopia \\ metii.teg@gmail.com
}

\begin{abstract}
Background: Immunophenotypic characterization of acute leukemia is an important clinical application of flow cytometry and has become a powerful tool contributing to proper diagnosis and classification. The aim of this study is to phenotype and classify acute leukemias by flow cytometry using commonly used markers for leukemia diagnosis.
\end{abstract}

Methods: A total of 40 pediatric and adult patients diagnosed with acute leukemia were evaluated by flow cytometry with 17 surface and cytoplasmic markers known to be useful in discriminating different types of acute leukemia. These results were compared with classification results using standard morphological criteria.

\begin{abstract}
Results: 21 of 40 patients (52.5\%) were classified as acute myeloblastic leukemia (AML) while 19 (47.5\%) were identified as acute lymphoblastic leukemia (ALL). Of all the ALL cases, 10 of the 19 (52.6\%) were B-ALL and $47.4 \%$ (9/19) were T-ALL based on flow cytometry. Markers of immaturity HLA-DR and CD34 antigens were co-expressed in $61 \%$ of AML cases and $33 \%$ of T-ALL cases, whereas CD34 was expressed in 50\% of the B-ALL cases. CMPO and CD13 were the most expressed markers of AML, CD19 and CCD79a were present in all cases of B-ALL, and cytoplasmic CD3 and CD7 were positive on most all T-ALL cases. Discrimination of AML from ALL patients by flow cytometry was $80 \%$ concordant with traditional morphology. Notable discrepancies occurred in cases where leukemia cells expressed markers for more than one lineage.
\end{abstract}

Conclusions: In the Ethiopia setting, flow cytometry represents a feasible and promising adjunctive diagnostic approach for acute leukemia.

\section{INTRODUCTION}

Immunophenotypic analysis of the reactivity of leukemic cells with monoclonal antibodies has proved to be useful in the diagnosis of acute leukemias. Flow cytometry offers a series of powerful tools for a precise definition of cell populations in bone marrow or peripheral blood specimens. Furthermore, by identifying sequential steps of cell maturation, flow cytometry can define different patterns of differentiation of individual lineages [1]. The flow was first shown to be relevant in the discrimination of acute lymphocytic and acute myeloid leukemia, particularly when the nature of the blasts cannot be defined by morphology and cytochemistry. Cases of "undifferentiated" acute leukemias include those with poorly differentiated myeloblasts (AML-MO) or those derived from early erythroid and megakaryocyte precursors [2]. Flow-based definition of acute lymphocytic leukemia as T lymphoid or B lymphoid, as well as establishing dual lineage in bi-phenotypic leukemia is valuable. Definition of maturation stage and finding possible aberrant antigens is what in turn serves for individual treatment monitoring and detection of residual disease [3]. 
Current modes of treatment for acute myeloblastic leukemia (AML) and acute lymphoblastic leukemia (ALL) are sufficiently different that an imprecise diagnosis can adversely affect prognosis. Newer therapeutic approaches have also increased the importance of assigning certain cases to the correct subtype of AML or ALL. In Ethiopia, Acute leukemia is one of the most serious public health problems. According to an earlier study by Shamebo $[4,5]$ in the 1990s in Tikur Anbessa (Black Lion) Hospital, a teaching and referral hospital in Addis Ababa, the commonest type of leukemia was chronic myeloid leukemia (CML) 57.8\%; acute leukemias and chronic lymphatic leukemia (CLL) accounted for $21.1 \%$ each. Of the acute leukemias, $53.3 \%$ were ALL while $46.7 \%$ were AML.

Currently, morphology and sometimes cytochemical techniques are the standards for diagnosis. Although cytochemical stains are essential to recognize the subtypes of AML, they are of limited use in differentiating the subtypes of ALL, thus limiting their application in a predominantly pediatric population, in which $85 \%$ to $90 \%$ of acute leukemia is of the lymphoid lineage. Moreover, cytomorphology and cytochemical stains utilize bone marrow samples, which is technically invasive and accurate examination of smears that also require technical skills not uniformly available in different hospitals and rural areas [6]. Thus, there is a need to introduce flow cytometry in the diagnosis of acute leukemias in Ethiopia. The aim of this study was to phenotype and classify acute leukemias by flow cytometry using commonly used markers for leukemia diagnosis and compare this methodology with traditional morphological diagnosis.

\section{METHODS}

\section{Study area and setting}

The study was conducted with patients with acute leukemia diagnosed at Tikur Anbessa Specialized Hospital (TASH) hematology unit. TASH is one of the largest specialized tertiary referral and teaching hospitals in the country with over 700 beds. The hematology unit of the internal medicine department is devoted to this. The data from the registration of the hematology unit shows that in 2014 and 2015, over 1177 leukemia patients were diagnosed, of which $43 \%$ were ALL, $17 \%$ $\mathrm{AML}, 29 \%$ chronic myelogenous leukemia, and $11 \%$ chronic lymphocyte leukemia. Morphological examination of peripheral blood and bone marrow aspiration are the routine diagnostic methods currently used in the hospital; occasionally, cytochemical staining techniques are also nous employed. A facility-based cross-sectional study was conducted to initiate the diagnostic utility of immunophenotypic analysis of acute leukemia by flow cytometry in Tikur Anbessa Specialized Hospital, from June to August 2016. Both adult and pediatric patients were diagnosed with acute leukemia and indicated morphological examination of peripheral blood and bone marrow aspiration. A total of 40 patients diagnosed with acute leukemia that fulfill the inclusion criteria were participants of this study.

\section{Sample collection}

A convenient sampling method was used, and patients who were newly diagnosed with acute leukemia, who had morphology analysis performed on peripheral blood and bone marrow aspirates, and who gave consent to participate in the study were included. Consent and assent were obtained from acute leukemia diagnosed patients using a standardized and structured format. Socio-demographic data and morphologic results of the peripheral blood and bone marrow aspirate smears of the study participants were collected from the patient medical record by the data collector. Peripheral blood was collected by the assigned nurses and delivered immediately to the principal investigator for flow cytometry analysis at the ALERT and AHRI Laboratory.

\section{Flow cytometry}

For immunophenotyping, peripheral blood (PB) samples were collected in EDTA tubes and immediately transported to the flow cytometry laboratory and processed and stained within 24 hrs. Flow cytometric analyses were done with a Becton Dickson FACSCalibur instrument (4-color) at ALERT/AHRI Laboratory. Different combinations of monoclonal antibodies (mAb) against the following antigens were used: cytoplasmic CD3 (cCD3), cytoplasmic CD79a (cCD79a), cytoplasmic MPO (cMPO), CD34, CD3, CD4, CD7, CD8, immunoglobulin kappa or lambda light chains, CD14, CD45, CD10, CD19, CD13, CD33, CD14, CD2, HLA-DR and CD117. Antibodies were purchased from Becton Dickinson and Agilent.

For surface antigen staining, 100 microliters of sample blood were stained with 5-20 microliters of fluorescently labeled monoclonal antibody (mAb) as appropriate and incubated in the dark at room temperature (RT) for 20 minutes, lysed with FACS lysing solution (Becton Dickinson), and incubated at room temperature for an additional $10 \mathrm{~min}$. After centrifugation at $400 \mathrm{x} g$, the tube supernatants were decanted, the cells were vigorously resuspended with phosphatebuffered saline (PBS), again centrifuged and the supernatant decanted, and the cells resuspended in an appropriate amount of PBS (typically $400 \mathrm{ul}$ ).

For detection of cytoplasmic antigens, peripheral blood samples were surface stained with 10 microliters of anti-CD45 PerCP-Cy5.5 and incubated in the dark at room temperature for 20 minutes and lysed lysing solution; PBS was added to a final volume of $4 \mathrm{cc}$, and cells centrifuged as above. After the supernatant was 
decanted, $500 \mu$ l of permeabilization reagent (BD Perm2 appropriately diluted) was added. After a 10-minute incubation at room temperature in the dark, cells were washed with PBS, 10 microliters of conjugated antibodies specific for cytoplasmic antigens were added, and the cells vortexed and incubated for $20 \mathrm{~min}$ in the dark. The cells were then washed after centrifugation at $800 \mathrm{x} g$ for 12 minutes, decanted, resuspended in $400 \mathrm{ul}$ of PBS, and acquired on the FACSCalibur.

Acquired cells were analyzed with the most appropriate blast gate using the combination of CD45 and side scatter. To assign molecular expression for categorical analysis, a marker was considered positive when at least $20 \%$ of the gated leukemia cells were positive.

All the quality control measures were undertaken before starting the procedure. BD FACSCalibur Instrument photomultiplier tube and compensation settings were performed by using control blood samples stained with leukogate (anti-CD45-FITC and CD14-PE) and monostained with anti-CD3-FITC, anti-CD56PE, anti-CD45 PerCP-Cy5.5, and anti-CD3 APC. Separate samples stained with surface or cytoplasmic isotype control antibodies were used to define gates that categorize a given marker as positive or negative. In addition, the dead cell stain 7-AAD was run as a separate sample for all patients to assure that dead cells did not substantially contribute to the phenotypic profile.

Leukemia was classified as AML, B-ALL, or T-ALL by flow cytometry based on the positive expression of at least two lineage-associated markers. Additional weight was given on marker expression with high known sensitivity (e.g., CD19 for B-ALL and CD7 for T-ALL), high specificity (e.g., CD117 for some $A M L$ ), or high sensitivity and specificity (e.g., cCD3 for T-ALL) [8].

\section{Data analysis}

Marker expression was defined as a percentage of leukemia gated cells above the threshold value defined by the negative isotype control tubes. Leukemia gates were defined by CD45 and perpendicular light scatter as defined above. Leukemia classification was based on criteria as described (8). SPSS version 20 was used for data entry and analysis. Concordance between flow and morphology methods in the diagnosis of AML and ALL was determined by summing the number of agreedupon cases divided by the total cases. The kappa statistic was used as an additional measure of concordance which corrects for random associations, with concordance considered "fair" when kappa value lies between 0.21 0.40, "moderate" between 0.41-0.60, "substantial" between $0.61-0.80$, and almost perfect when kappa is 0.81-1.00 [7].

\section{RESULTS}

\section{Gating of leukemic cells and definition of marker positive and negative cells}

Figure 1 depicts plots defining multiple parameters after staining of leukemia containing peripheral blood. Leukemia cells, outlined in the white dotted circle polygon, typically exhibited reduced CD45 expression compared to normal leukocytes. It depicts CD45 expression ( $\mathrm{x}$-axis) as a function of multiple other markers (e.g., CD13, CD10, and HLA-DR in this example); thus, it can readily reveal the expression of such markers on leukemia cells (white dotted circles), as opposed to normal peripheral blood cells with higher CD45 density, visualized in the middle panels, Figure 1. Gating (electronically selecting) leukemia cells based on CD45 and SSC, allowing for the visualization of the marker of interest on the leukemia cells exclusive of nonleukemia cells.

The definition of cut-off values or regions to define marker positive and negative cells was done with an independent control tube containing fluorochromeconjugated antibodies without reactivity to human cells. Such stained cells typically exhibit a small shift in nonspecific staining relative to unstained cells and thus serve as a more suitable means to define cutoff values. Figure 2 depicts such control antibody staining by antiCD45 stained leukemia cells. By convention, a region is defined, e.g., R3 in the lower-left plot, encompassing nearly all the control-stained cells, and an adjacent region, e.g., R2, which includes all values along the $y$-axis higher than the first region (R3). These region settings are applied to all stained samples within the experiment, and thus define the marker negative population (e.g., R3) and marker positive population (e.g., R2) for each marker in the experiment. Typically, separate positive and negative regions are defined for each fluorochrome to be used in combination with CD45. Since all other marker antibodies were conjugated to the same fluorochromes used in the control sample, the positive and negative regions defined in this control will serve as adequate controls for all other markerantibody conjugates used in the experiment.

\section{Leukemia case summary and demographics}

A total of 40 acute leukemia cases were phenotyped by flow cytometry (Table 1 ). Twenty-one cases were classified as acute myelogenous leukemia (AML) and 19 as acute lymphocytic leukemia (ALL). Of the ALL cases, there were 10 (52.6\%) identified as B lineage leukemia cells (B-ALL), and 9 (47.5\%) defined as $T$ lineage cells (T-ALL). With respect to gender frequency, females represented $47.5 \%$ and males $52.5 \%$ of the cases. A total of $25 \%$ of the patients were less than 18 years of age while $75 \%$ were 18 and above. 
Figure 1.

Definition of leukemia cells by expression of CD45 and side scatter and phenotyping of such gated cells
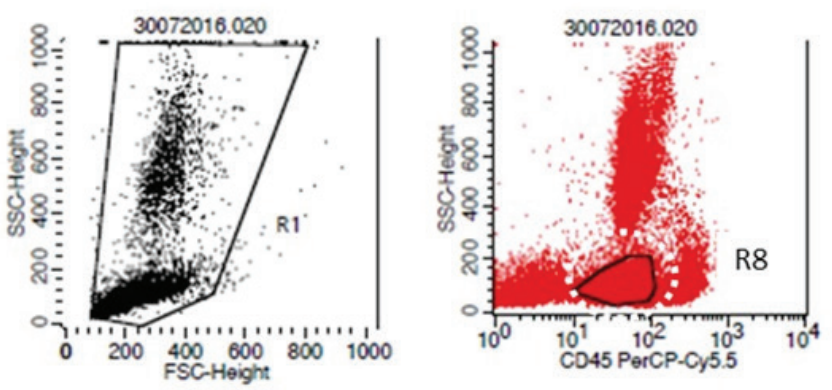

$-R 1$ is all estimated live white

cells

$-R 8$ is estimated leukemia region -Second row of three panels are gated on R1

-Third row of panels gated on $\mathrm{R} 1$ and $\mathrm{R} 8$
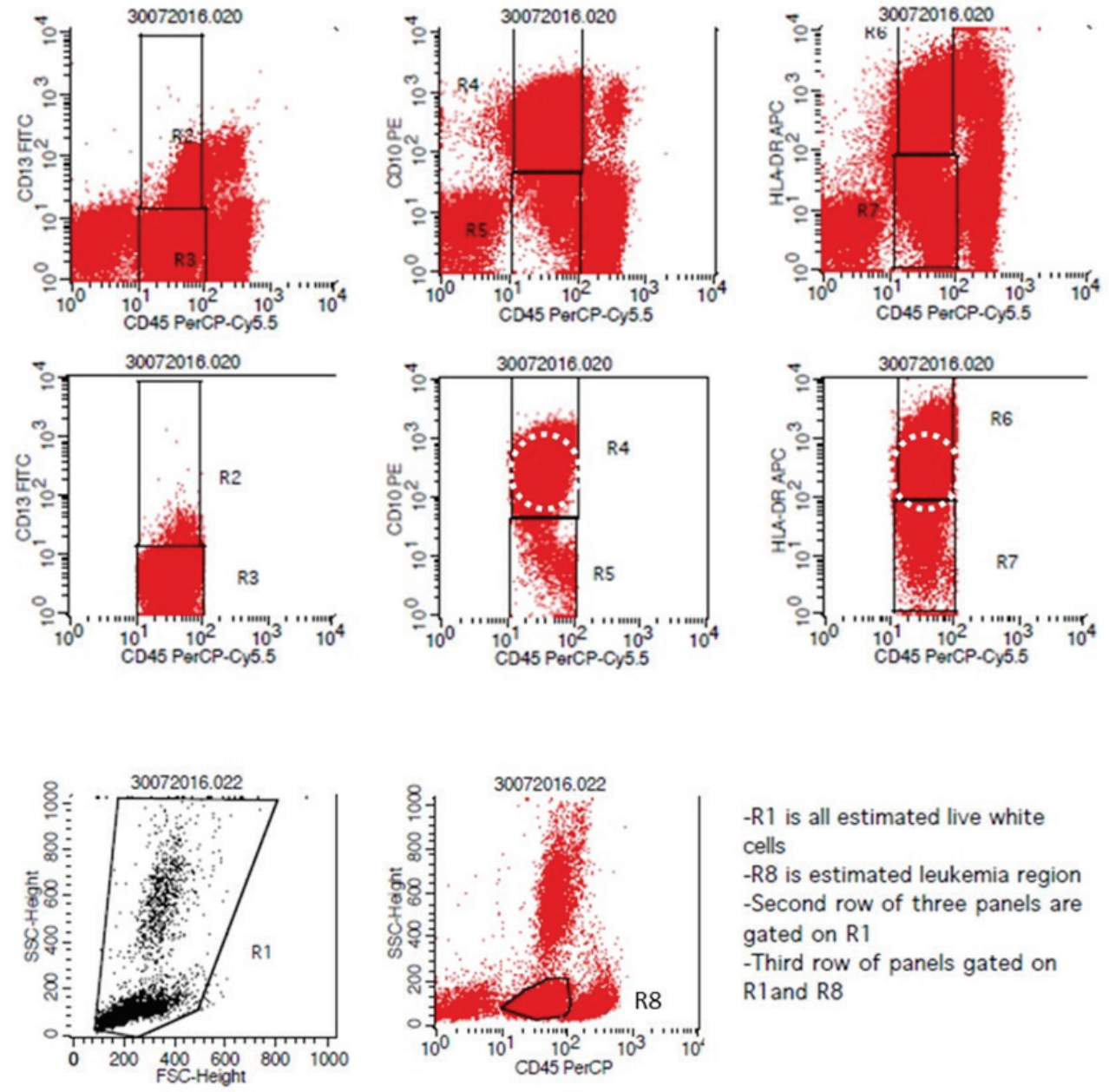

$-R 1$ is all estimated live white cells

$-R 8$ is estimated leukemia region -Second row of three panels are gated on R1

-Third row of panels gated on

$\mathrm{R} 1$ and $\mathrm{R} 8$
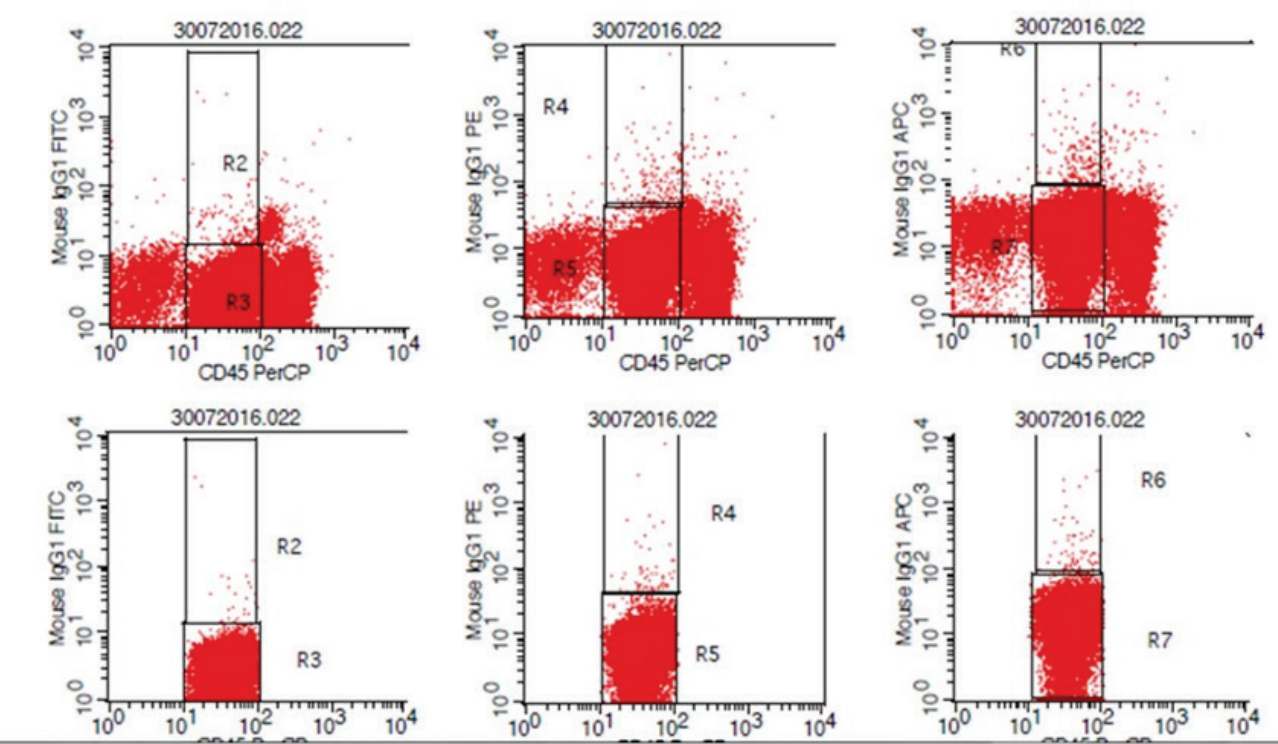

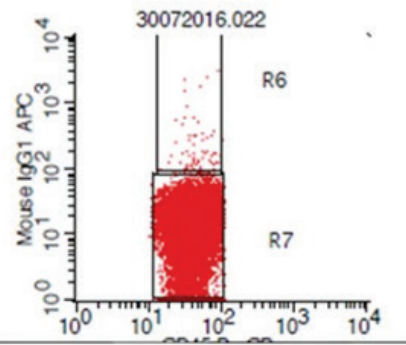

Figure 2.

Creation of regions to define marker positive and negative cells using IgG control antibodies 


\section{Phenotypic analysis of AML, B-ALL, and T-ALL}

Myeloid markers evaluated included CD33, CD13, CD117, and cMPO. A given marker expressed by $>20 \%$ of the gated leukemia cells were defined as positive and the percentage of positive cases among all AML for each marker determined. CMPO, the most commonly expressed marker, was expressed in 20 of 21 (95\%) AML cases, CD13 was expressed in 19 of 21 (90\%) of the cases, and CD33 and CD117 in 18 (85.7\%) and 16 (76\%) cases, respectively. The total marker positive cells were also defined for each leukemia case and each marker, and the mean and standard error for each marker determined among the entire set of AML cases. The mean \% cMPO, $\% \mathrm{CD} 13, \% \mathrm{CD} 33$, and \% CD117 positive cells was 86.6 $\pm 20.8,60 \pm 30.0,65.7 \pm 32$ and $53 \pm 32.7$, respectively (Table 2). Among acute leukemia cases classified as B-ALL, CCD79a and CD19 were expressed by at least $20 \%$ of leukemic cells for all cases, whereas CD10 was positive in $80 \%$ of the cases. The \% mean + standard deviation for CCD79a, CD19, and CD10 for the B-ALL group was $82.2 \pm 16.2$ and $77.4 \pm 25.1,60.2 \pm 35.9$, respectively. (Table 2). All cases of T-ALL were positive for cytoplasmic CD3 with a mean of $86.9 \pm 11.7 \%$ positive cells; CD7 was positive in 7 of 8 cases, and among all cases of T-ALL, the mean $\%$ CD7 positive cells was $71.9 \pm 35.8$. Surface CD3 expression was more variable among T-ALL cells, positive in only $66 \%$ of the cases, and CD4 and CD8 expression were similarly variable, positive in 55\% and $44 \%$ of the T-ALL cases, respectively. The mean $\%$ surface CD3, CD4, and CD8 positive cells among the 10 cases of T-ALL was $40.4 \pm 27.7,24.3 \pm 25.4$, and $25.1 \pm$ 23.6, respectively. $C D 2$, a marker normally expressed by nearly all mature $T$ cells and other non- $T$ cells, was positive in 5 of 7 cases (71\%), and the mean among all cases defined with this marker was $59.1 \pm 36.4 \%$ positive cells (Table 2).

\section{Expression of markers of immaturity}

We evaluated markers of immaturity including CD34, known to be present on early progenitor cells within AML, B-ALL, and T-ALL lineages, CD117, expressed principally on early AML lineage cells; and HLA-DR, detectable on early AML and T-ALL, but constitutively expressed on all B-ALL lineage cells. Among AML cases, CD34 was expressed in 13 of 21 cases (62\%), whereas HLA-DR was positive in 14 of 21 cases (66.7\%) (Table 3). All 13 of the patients positive for CD34 were also positive for HLA-DR, whereas 7 of 21 (33\%) were negative for both markers, and 1 positive for HLA-DR but CD34 negative. Moreover, CD117 was simultaneously expressed in 11 of the 13 HLA-DR+CD34+ cases, and an additional 5 AML cases which were HLA-DR-CD34In the B-ALL group of leukemias, 5 of 10 (50\%) exhibited CD34 expression, and, as expected, all 5 co-expressed HLA-DR (Table 3). In the T-ALL group, 1 case was
CD34+HLA-DR+, 3 cases were CD34-HLA-DR-, and 5 additional cases expressed either CD34 or HLA-DR (not shown in Table 3). One T-ALL leukemia expressed CD117 without HLA-DR or CD34 (Table 3).

\section{Aberrant antigen expression}

In our study, aberrant expression of myeloid antigens was seen in some ALL cases (Table 2). Within the B-ALL group, CD33, CD13, and CMPO were observed in $10 \%$, $40 \%$, and $60 \%$ of the cases, respectively. In none of the cases was more than one myeloid marker detected. 2 of 9 cases of T-ALL displayed CMPO, 1 case was positive for $\mathrm{CD} 19$, and 1 positive for CD117. $44 \%$ of T-ALL cases showed CD10 expression and these were all negative for CD19. Out of 9 cases of T-ALL, two expressed CD33 and CD13. Aberrant expression of lymphoid antigens was also seen among AML leukemias (Table 2). One case was positive for the $B$ lineage marker CD19, and 4 of 21 (19\%) were positive for the B lineage marker CCD79a.

\section{Agreement of acute leukemia classification by morphology vs flow cytometry}

The traditional FAB classification of ALL and AML is based on morphology and cytochemical staining of blasts, the recent classification schemes proposed by WHO requires the additional evaluation of the leukemic blasts by flow cytometry and molecular analysis of chromosomal rearrangements and mutations, while the EGIL classification has proposed that acute lymphocytic leukemia subtypes be classified based on immunophenotype alone. In order to provide perspective on the flow cytometry-based classifications for the leukemias in our study, we compared them with the classification provided by pathologists at Tikur Anbessa Specialized Hospital after a review of peripheral blood and bone marrow aspirate smears. Of the 40 leukemia cases we phenotyped by flow cytometry, 23 were classified as $A M L, 15$ as ALL, and two as indeterminate by morphology. This contrasted with the flow classification of $21 \mathrm{AML}$ and $19 \mathrm{ALL}$ (i.e., either B-ALL or T-ALL). As depicted in Table 4, among the 21 cases of AML classified by flow cytometry, 19 (90.4\%) were concordantly diagnosed as such by morphology, while 2 were classified as ALL. Conversely of the 23 cases of AML by morphology, 19 were classified similarly by flow cytometry. Of the 15 cases of ALL defined by morphology, 13 were defined as such by flow cytometry, while 2 were classified as AML by flow phenotyping. Finally, the two indeterminant cases by morphology were typed as ALL by flow cytometry analysis. The degree of statistical concordance is summarized in Table $\mathbf{5}$, illustrating an overall $80 \%$ agreement between morphology and flow cytometry classification, and a kappa statistic of $0.614(p=0.0000)$, considered to be "substantial agreement" [7]. 
Table 1. Demographic of acute leukemia patients at Tikur Anbessa Specialized Hospital, Addis Ababa, June 2016 to August $2016(n=40)$

\begin{tabular}{lcc}
\hline Variable & Number & Percent (\%) \\
\hline Male & 21 & 52.5 \\
Female & 19 & 47.5 \\
Age $<18$ years old & 10 & 25 \\
Age 18 and above & 30 & 75.0 \\
ALL classification & 19 & 47.5 \\
AML classification & 21 & 52.5 \\
\hline
\end{tabular}

ALL, acute lymphocytic leukmemia; AML,acute myelocytic leukemia; classification as defined flow cytometry

Table 2. Expression of phenotypic markers by different categories of leukemia

\begin{tabular}{|c|c|c|c|c|c|c|c|c|c|}
\hline & $A M L$ & & & B-ALL & & & T- ALL & & \\
\hline $\begin{array}{l}\text { CD } \\
\text { Marker }\end{array}$ & $\begin{array}{l}\text { \% Pos } \\
\text { Cases** } \\
(n=21)\end{array}$ & Mean & SD & $\begin{array}{l}\% \text { Pos } \\
\text { Cases * } \\
(n=10)\end{array}$ & Mean\# & SD & $\begin{array}{l}\% \text { Pos } \\
\text { Cases } * \\
(n=9)\end{array}$ & Mean & SD \\
\hline CD3 & 0 & 2.0 & 2.8 & 0 & 2.0 & 2.3 & $6(66)$ & 40.4 & 27.7 \\
\hline CD4 & $3(14)$ & 12.1 & 14.8 & 0 & 3.6 & 3.5 & $5(55)$ & 24.3 & 25.4 \\
\hline CD8 & 0 & 1.4 & 2.7 & 0 & 1.5 & 1.1 & $4(44)$ & 25.1 & 25.6 \\
\hline CD19 & $1(4.7)$ & 5.7 & 9.2 & $10(100)$ & 77.4 & 25.1 & $1(11)$ & 14.6 & 26.8 \\
\hline cCD79a & 4 (19) & 12.7 & 19.8 & $10(100)$ & 82.2 & 16.2 & 0 & 4.6 & 3.8 \\
\hline cCD3 & $0 \%$ & 1.3 & 1.9 & 0 & 4.5 & 5.6 & $9(100)$ & 86.9 & 11.7 \\
\hline CD10 & $3(14.2)$ & 9.8 & 21.6 & $8(80)$ & 60.2 & 35.9 & $4(44)$ & 40.4 & 44.7 \\
\hline CD33 & 18 (85.7) & 65.7 & 32 & $1(10)$ & 7.4 & 8.8 & $2(22)$ & 22.6 & 40.7 \\
\hline CD13 & $19(90)$ & 60 & 30.0 & $4(40)$ & 9.4 & 11.8 & $2(22)$ & 19.7 & 34.1 \\
\hline cMPO & 20 (95) & 86.6 & 20.8 & $6(60)$ & 45.6 & 39.9 & $2(22)$ & 12.7 & 18.1 \\
\hline CD117 & $16(76)$ & 53 & 32.7 & 0 & 0.41 & 0.5 & $1(11)$ & 3.5 & 7.7 \\
\hline CD2 & - & - & - & 0* & $2.6^{*}$ & $1.3^{*}$ & $5(71) \ddagger$ & $59.1 \ddagger$ & $36.4 \ddagger$ \\
\hline CD7 & - & - & - & $1(10)$ & 11.2 & 16 & $7(87)+$ & $71.9+$ & $35.8^{\dagger}$ \\
\hline CD34 & $13(62)$ & 52 & 42.0 & $5(50)$ & 35 & 41.8 & $3(33)$ & 15.6 & 22.9 \\
\hline HLA-DR & $14(66.6)$ & 54 & 43.2 & $10(100)$ & 86 & 14.8 & $3(33)$ & 13.1 & 18.5 \\
\hline
\end{tabular}

\#The percentage of positive cells determined for each marker and each case, and the mean and standard deviation of marker positive cells determined for the entire group of leukemia cases

* A leukemia case was scored positive for a given marker if more than $20 \%$ of the leukemia cells were positive for that marker $* \mathrm{n}=6, \ddagger \mathrm{n}=7,+\mathrm{n}=8$

Table 3. Co-expression of HLA-DR, CD34 and/or CD117 in acute leukemia's

\begin{tabular}{llll}
\hline CD Markers & $\begin{array}{l}\text { AML } \\
(\mathbf{n}=\mathbf{2 1})\end{array}$ & $\begin{array}{l}\text { B-ALL } \\
(\mathbf{n}=\mathbf{1 0})\end{array}$ & $\begin{array}{l}\text { T-ALL } \\
(\mathbf{n}=\mathbf{9})\end{array}$ \\
\hline CD34+/HLADR+ & $13(62 \%)$ & $5(50 \%)$ & $1(11 \%)$ \\
CD34-/HLADR- & $8(38 \%)$ & $0(0 \%)$ & $3(33 \%)$ \\
CD34+/HLA-DR+/CD117+ & $11(52 \%)$ & $0(0 \%)$ & $0(0 \%)$ \\
CD34-HLA-DR-/CD117+ & $5(24 \%)$ & $0(0 \%)$ & $1(11 \%)$
\end{tabular}


Table 4. Agreement of acute leukemia classification by morphology vs flow cytometry

\begin{tabular}{lllll}
\hline & & \multicolumn{3}{l}{ Flow Cytometry } \\
& Classification* & AML & ALL & Total \\
\hline Morphology & AML & 19 & 4 & 23 \\
& ALL & 2 & 13 & 15 \\
& Inconclusive & 0 & 2 & 2 \\
& Total & 21 & 19 & 40 \\
\hline
\end{tabular}

*The degree of association between morphology and flow cytometry was evaluated by chi-square testing (Pearsons Chi-square $=14.6$ Person, $p=0.001$ )

\section{Flow cytometry phenotype among leukemias cases with discordant classification}

To explore possible reason(s) for discrepancies between morphology and flow evaluation of leukemia, we considered the possibility that discordant cases may have preferentially occurred in leukemia which expressed markers of more than one lineage. Table 6 summarizes cell surface phenotype of some of the discordantly classified leukemias stratified by markers that were interpreted to be lineage-specific (and hence defined the flow-based classification) and those interpreted to be aberrantly expressed. Two cases of T-ALL were defined by flow cytometry which had been classified as $A M L$ by morphology. Both cases were strongly positive for cytoplasmic CD3 and variably expressed other $\mathrm{T}$ cell markers. However, both leukemias also expressed some myeloid antigens including CMPO, CD33, and/or CD13. According to the literature, myeloid antigen expression is commonly observed among cases of T-ALL, but conversely, CCD3 expression is uncommon among $\mathrm{AML}$ cases (8). Consequently, the lineage was assigned to $T$ lymphoid rather than myeloid in these cases. Similarly, another case occurred in which the acute leukemia was diagnosed as AML by morphology, but B-ALL based on flow, and in this case, myeloid markers CD13 and CD33 were also present, the final flow classification again based on the likely lineage origin after consultation of the literature [8].

\section{DISCUSSION}

The capacity to quantitative numerous unique molecules on individual cells by flow cytometry is ideal for the study of leukemic cells. Such immunophenotyping has become an important and sensitive tool contributing, with clinical, morphological, cytochemical, and cytogenetic analyses, to the classification, prognosis, and disease monitoring of acute leukemia [9]. The classification into B- and T-lineage ALL is important for risk stratification and therapy of the patients [10]. Based on flow cytometry phenotyping, 40 blood samples of acute leukemia were classified into 21 AML (52.5\%) and $19 \mathrm{ALL}$ (47.5\%) cases. This distribution was similar to a previous study in Ethiopia based on morphology analysis by Shamebo et al. [5], which demonstrated 46.3\% AML and 53.7\% ALL among acute leukemia cases during a 10-year period from 1982 to 1992. However, both studies have been based on patients diagnosed in a tertiary care referral center, and hence can only be considered approximate estimates for the incidence of these leukemia's nationwide. ALL cases were further subclassified into B-cell (10 cases) and T-cell (9 cases) ALL. Our results were also comparable to a study by Salem et al. in Egypt though they observed a higher fraction of AML (69\%) than ALL, and a higher proportion of B-ALL (75\%) relative to T-ALL among ALL cases (11). Among the markers tested in the AML cases, CMPO was nearly universally expressed with CD13, CD33, and CD117 also expressed in the majority of patients. These phenotypic findings are quite similar to those reported by Salem et al. (11). Our results are also consistent with Paredes-Aguilera et al. [12] who concluded that in comparison with $\mathrm{CD} 13, \mathrm{CD} 14$, and $\mathrm{CD} 33$, cMPO was the most sensitive marker for AML.

All cases of B-ALL expressed CCD79a and CD19. CD10 was variably expressed ( $80 \%$ positive). Of note, according to the EGIL classification system, CD10 is not expressed

Table 5. Cell surface phenotype among some acute leukemia's cases with discordant classification

\begin{tabular}{lllll}
\hline PM result & BM result & FCA result & Lineage specific phenotype & Aberrant expression \\
\hline$A L L$ & Inconclusive & B-ALL & $C D 19, C C D 79 a, C D 10, H L A-D R$ & cMPO \\
ALL & Inconclusive & AML & $C D 33, C D 13, C D 117, C M P O, C D 34, H L A-D R$ & No \\
$A M L$ & $A M L$ & T-ALL & $C D 8, C C D 3, C D 2, C D 7$ & CMPO \\
$A M L$ & $A M L$ & T-ALL & $C D 3, C C D 3, C D 7, C D 2, C D 34$ & $C D 10, C D 33, C D 13$ \\
$A M L$ & $A M L$ & $B-A L L$ & $C D 19, C C D 79, C D 10, C D 34, H L A-D R$ & $C D 13, C D 33$
\end{tabular}

ALL, acute lymphocytic leukmemia; AML,acute myelocytic leukemia; BM, bone marrow; FCA, flow cytomettry analysis 
by the most immature B-ALL cells (B1 or pro B cells), but it is expressed by B-ALL at intermediate stages (B2/ common $B$ cells or B3/pre-B cells). CD10 expression is reduced in the most mature EGIL stage (B4). The latter commonly express surface Immunoglobulin (Kappa and Lambda) which was uniformly negative in our cases, suggesting that, at least by the EGIL classification, our B-ALL cases all represented immature B cells (B1, B2, and B3) (13). Our findings are quite consistent with those of Zahid Kaleem et al. [14] as well as Salem et al. [11]. Paredes-Aguilera et al. [12] compared the sensitivity of B lineage markers cCD79a, cyCD22, CD19, CD20, and CD22 in their study of 74 cases of B-ALL and showed that CCD79a has $100 \%$ sensitivity and $80 \%$ specificity followed by $\mathrm{CCD} 22$, which showed $97 \%$ sensitivity and $88 \%$ specificity. They concluded that these are highly sensitive markers for B-cell ALL cases. Aberrant expression of myeloid antigens, CD33 (10\%), CD13 (40\%), and cMPO $(60 \%)$ were seen in some cases of B-ALL. The study by Salem et al. [11] also observed aberrant expression of CD13 and CD33 myeloid markers among B-ALL cases, but markers CMPO and CD117 were rarely expressed.

All cases classified as T-ALL were positive for cytoplasmic CD3. In addition, the $\mathrm{T}$ cell marker CD7 was observed in all but one case. Surface CD3 was observed in only $66 \%$ of the cases, an expected finding of immature $T$ lineage cells which have expressed CD3 but incomplete production T-cell receptor proteins which normally associate in a complex with CD3 and are required for $\mathrm{CD} 3$ to be expressed on the surface. CD4 (55\%) and CD8 (44\%) were variably expressed in T-ALL. Similarly, studies by Salem et al. [11] and Zahid Kaleem et al. [14] showed universal expression of cytoplasmic CD3, high CD7, but variable percentages of surface CD3, CD4, and CD8. In our study, the aberrant expression on T-ALL was seen for myeloid markers CD33 (22\%), CD117 (11\%), CD13 (22\%), and cMPO (22\%), whereas B cell markers CD10 (44\%) and CD19 (11\%) were inappropriately expressed. The study by Salem et al. [11] and others observed aberrant expression of CD10 ranging from $19-43 \%$ and CD19 varying from 0 to $2 \%$.

Expression of HLA-DR and CD34 was seen in about two-thirds of patients with AML, with nearly all cases either co-expressing these two immature markers or expressing neither molecule. The study by Salem et al. [11] exhibited, among non-acute promyelocytic leukemia (FAB M3 AML) cases, similar CD34 but higher HLA-DR. Most of the cases of AML in their study were classified as either M1 or M2 AML leukemia, which would be expected to have high CD34 and HLA-DR. Although we did not classify AML cases by morphology according to the FAB system, the predominance of CD34 and HLA-DR in our cohort would be consistent with a high fraction of $M 0, M 1$, or $M 2$ cases of AML. Further work in Ethiopia will be needed to determine more precisely $F A B$ classification in comparison with flow cytometry. About half of the B-ALL cases were positive for CD34. HLA-DR is expressed at all stages of $B$ cell maturation, and as expected, all of our B-ALL cases were HLA-DR positive. These findings on a limited number of B-ALL cases concur with those of Zahid Kaleem et al. [14] who observed about $70 \%$ of B-ALL expressed CD34 and virtually all were positive for HLA-DR. Only about a third of T-ALL cases were positive for both HLA-DR and CD34, suggesting that these represent very immature forms of T-ALL.

In general, we observed about $80 \%$ agreement between flow cytometry analysis and cell morphology as determined by evaluation of peripheral blood and bone marrow aspirate smears. Others have reported similar or higher concordance. Patel et al. [15] showed a strong concordance of $76 \%$ between flow cytometry and bone marrow morphology in acute leukemia cases in Kenya. In contrast to these studies, Belurkar et al. [16] observed a concordance rate as high as $86 \%$ between morphologic/cytochemical diagnosis and flow cytometric diagnosis among 50 cases of leukemia, and Khalil et al. [17] reported $97 \%$ concordance with immunophenotyping and the combination of morphology and cytochemistry. Importantly, the latter two studies combined morphology with cytochemistry in an acute leukemia diagnosis, and no cytochemistry was done in either our study or the Kenyan study. Cytochemistry is particularly useful at specifically identifying acute myeloid leukemia.

Despite the relatively large number of markers used in this study, many more markers have been used in many leukemia phenotyping studies [8]. The study would be improved by parallel analysis by cytochemistry, cytogenetic, or molecular analyses, but these methodologies were either unavailable or not yet developed in the country. Markers for AML leukemias M6 (erythroleukemia) or M7 (megakaryoblastic leukemia) were not included; hence, such leukemias, if present, may have been missed. More extensive patient clinical information was not captured, and the study ideally should be performed with much larger sample size. Finally, an important disadvantage of flow cytometry is the cost and required laboratory expertise in the setting of resource-limited countries. It is essential, therefore, to define in which subset of cases flow cytometry (and other modalities) should be considered as an adjunctive cost-effective diagnostic test beyond what is provided by standard morphology. Studies to address these issues are underway.

\section{CONCLUSIONS}

In general, there was good agreement between flow cytometry and morphology in the discrimination of myeloid from lymphoid leukemias. The ability of flow 
cytometry to confirm morphological diagnoses, to provide an alternative when morphology results are indeterminant, and to define lymphoid leukemia suggests more precisely it can represent a valuable supplementary methodology for clinical management of leukemia in this country.

\section{DECLARATIONS}

\section{Competing of Interest}

The author declares there are no known competing interests associated with this publication.

\section{Ethics Approval}

The study was approved by the institutional review committee of AHRI-ALERT ethics review board. The study approval no. was PO24/15. The written consent for all study participants form was ethically approved by the AHRI-ALERT Research Ethics Committees.

\section{Funding Source}

This study was funded by Armauer Hansen Research Institute. The funders have no role in the design of the study, data collection, analysis, interpretation of data, and in writing the manuscript.

\section{Acknowledgment}

The authors gratefully thank Armauer Hansen Research Institute and Addis Ababa University, School of Medicine, Department of Hematology and Pediatric for all supports provided.

\section{REFERENCES}

1. Del Vecchio L, Brando B, Lanza F, et al. Recommended reporting format for flow cytometry diagnosis of acute leukemia. Haematologica. 2004;89(5):594-8.

2. Rowan R, Bain BJ, England JM, et al. Immunophenotyping in the diagnosis of acute leukemias. J Clin Pathol. 1994;47:777-81.

3. Wozniak J, Kopec-Szlezak J. Standard immunophenotyping of leukemia cells in acute myeloid leukemia (AML). Centr Eur J Immunol. 2008;33(1):24-32.

4. Shamebo M. Acute leukaemias in adult Ethiopians in a teaching hospital. Ethiop Med J. 1994;32(1):17-25.

5. Shamebo M. Leukaemia in adult Ethiopians. Ethiop Med J. 1990;28(1):31-7.

6. Mhawech P, Buffone GJ, Khan SP, Gresik MV. Cytochemical staining and flow cytometry methods applied to the diagnosis of acute leukemia in the pediatric population: an assessment of relative usefulness. J Pediatr Hematol Oncol. 2001;23(2):89-92.
7. Viera AJ, Garrett JM. Understanding interobserver agreement: the kappa statistic. Fam Med. 2005;37(5):360-3.

8. Ortolani C. Flow cytometry of hematological malignancies. 1st ed. Chicester, West Sussex, UK: Willey-Blackwell Publishing; 2011.

9. Garand R, Robillard N. Immunophenotypic characterization of acute leukemias and chronic lymphoproliferative disorders: practical recommendations and classifications. Hematol Cell Ther. 1996;38(6):471-86.

10. Van Dongen J, Macintyre E, Gabert J, et al. Standardized RT-PCR analysis of fusion gene transcripts from chromosome aberrations in acute leukemia for detection of minimal residual disease Report of the BIOMED-I Concerted Action: Investigation of minimal residual disease in acute leukemia. Leukemia. 1999;13:1901-28.

11. Salem DA, El-Aziz SMA. Flowcytometric immunophenotypic profile of acute leukemia: mansoura experience. Indian J Hematol Blood Transfus. 2012;28(2):89-96.

12. Paredes-Aguilera R, Romero-Guzman L, LopezSantiago N, et al. Flow cytometric analysis of cellsurface and intracellular antigens in the diagnosis of acute leukemia. Am J Hematol. 2001;68(2):69-74.

13. Peters JM, Ansari MQ. Multiparameter flow cytometry in the diagnosis and management of acute leukemia. Arch Pathol Lab Med. 2011;135(1):44-54.

14. Kaleem Z, Crawford E, Pathan MH, Jasper L, Covinsky $M A$, Johnson LR, et al. Flow cytometric analysis of acute leukemias: diagnostic utility and critical analysis of data. Arc Pathol Lab Med. 2003;127(1):42-8.

15. Patel K, Lotodo T, Njuguna F, et al. Use of flow cytometry immunophenotyping for diagnosis of acute leukemia at Moi Teaching and Referral Hospital, Eldoret, Kenya. American Scientific Research Journal for Engineering, Technology, and Sciences (ASRJETS). 2015;13(1):72-80.

16. Belurkar S, Mantravadi H, Manohar C, Kurien A. Correlation of morphologic and cytochemical diagnosis with flowcytometric analysis in acute leukemia. J Cancer Res Ther. 2013;9(1):71-9.

17. Khalil S, Jackson J, Pyle R, Robichaud $M$. Immunophenotyping of acute leukemia at King Faisal Specialist Hospital and Research Centre. Ann Saudi Med. 1995;15(2):137-9. 